\title{
Birkhoff-von Neumann graphs that are PM-compact
}

\author{
Marcelo H. de Carvalho ${ }^{a *} \quad$ Nishad Kothari $^{b \dagger} \quad$ Xiumei Wang ${ }^{c \ddagger}$ \\ Yixun $\operatorname{Lin}^{c}$ \\ ${ }^{a}$ Institute of Computing, UFMS, Campo Grande, Brazil \\ ${ }^{b}$ Faculty of Mathematics, University of Vienna, Austria \\ ${ }^{c}$ School of Mathematics and Statistics, Zhengzhou University, Zhengzhou, China
}

26 June, 2019

\begin{abstract}
A well-studied geometric object in combinatorial optimization is the perfect matching polytope of a graph $G$ - the convex hull of the incidence vectors of all perfect matchings of $G$. In any investigation concerning the perfect matching polytope, one may assume that $G$ is matching covered - that is, $G$ is a connected graph (of order at least two) and each edge of $G$ lies in some perfect matching.

A graph $G$ is Birkhoff-von Neumann if its perfect matching polytope is characterized solely by non-negativity and degree constraints. A result of Balas (1981) implies that $G$ is Birkhoff-von Neumann if and only if $G$ does not contain a pair of vertexdisjoint odd cycles $\left(C_{1}, C_{2}\right)$ such that $G-V\left(C_{1}\right)-V\left(C_{2}\right)$ has a perfect matching. It follows immediately that the corresponding decision problem is in co- $\mathcal{N} \mathcal{P}$. However, it is not known to be in $\mathcal{N} \mathcal{P}$. The problem is in $\mathcal{P}$ if the input graph is planar due to a result of Carvalho, Lucchesi and Murty (2004). More recently, these authors, along with Kothari (2017), have shown that this problem is equivalent to the seemingly unrelated problem of deciding whether a given graph is $\overline{C_{6}}$-free.

The combinatorial diameter of a polytope is the diameter of its 1-skeleton graph. A graph $G$ is PM-compact if the combinatorial diameter of its perfect matching polytope equals one. Independent results of Balinski and Russakoff (1974), and of Chvátal (1975), imply that $G$ is PM-compact if and only if $G$ does not contain a pair of vertexdisjoint even cycles $\left(C_{1}, C_{2}\right)$ such that $G-V\left(C_{1}\right)-V\left(C_{2}\right)$ has a perfect matching. Once again the corresponding decision problem is in co- $\mathcal{N} \mathcal{P}$, but it is not known to be in $\mathcal{N} \mathcal{P}$. The problem is in $\mathcal{P}$ if the input graph is bipartite or is near-bipartite - due to a result of Wang, Lin, Carvalho, Lucchesi, Sanjith and Little (2013).
\end{abstract}

\footnotetext{
*Supported by Fundect-MS and CNPq.

†Supported by Austrian Science Foundation FWF (START Y463) and by FAPESP Brazil (2018/04679-1).

${ }^{\ddagger}$ Supported by the National Natural Science Foundation of China (Nos. 11571323 and 11801526).
} 
In this paper, we consider the "intersection" of the aforementioned problems. We give an alternative description of matching covered graphs that are Birkhoff-von Neumann as well as PM-compact; our description implies that the corresponding decision problem is in $\mathcal{P}$.

\section{Contents}

1 The perfect matching polytope

1.1 Birkhoff-von Neumann graphs . . . . . . . . . . . . . . . 3

1.2 PM-compact graphs . . . . . . . . . . . . . . . . . 3

1.3 Conformal bicycles . . . . . . . . . . . . . . . . . . . 3

1.4 Main result ......................... 4

2 Matching covered graphs 6

2.1 Tight cut decomposition .................... 7

2.2 Solid graphs . . . . . . . . . . . . . . . . . . . . 7

2.3 Proof of Theorem $1.9 \ldots \ldots \ldots \ldots$

3 Bricks $\quad 11$

3.1 Strictly thin edges . . . . . . . . . . . . . . . . . 11

3.2 Adding an edge . . . . . . . . . . . . . . . . . . . . . 13

3.3 Index one . . . . . . . . . . . . . . . . . . . . . 14

3.4 Index two . . . . . . . . . . . . . . . . . . . 17

3.5 Index three . . . . . . . . . . . . . . . . . . . 18

3.6 Proof of Theorem $2.4 \ldots \ldots \ldots \ldots \ldots$

3.7 An infinite family . . . . . . . . . . . . . . . . 24

\section{The perfect matching polytope}

Graphs considered in this paper are loopless; however, they may have multiple edges (joining any two vertices). A graph is simple if it is devoid of multiple edges. For a graph $G:=(V, E)$, we use $\mathbb{R}^{E}$ to denote the set of real vectors whose coordinates are indexed by the edges of $G$. The perfect matching polytope of $G$, denoted by $\mathcal{P}$ oly $(G)$, is the convex hull of the incidence vectors of all perfect matchings of $G$.

For a set $S \subseteq V$, the cut of $S$, denoted by $\partial(S)$, is the set of edges of $G$ that have one end in $S$, and the other end in $\bar{S}:=V(G)-S$. We refer to $S$ and $\bar{S}$ as the shores of the cut $\partial(S)$. A cut is trivial if either shore is a singleton. For a vertex $v$ of $G$, we simplify the notation $\partial(\{v\})$ to $\partial(v)$. For a vector $x \in \mathbb{R}^{E}$ and a set $F \subseteq E$, we use $x(F)$ to denote $\sum_{e \in F} x(e)$. 


\subsection{Birkhoff-von Neumann graphs}

For a graph $G:=(V, E)$, a vector $x \in \mathbb{R}^{E}$ is 1-regular if $x(\partial(v))=1$ for each vertex $v$. Since the incidence vector corresponding to any perfect matching is non-negative and 1-regular, it follows that each vector in $\mathcal{P}$ oly $(G)$ is non-negative and 1-regular. In the case of bipartite graphs, this obvious necessary condition is in fact sufficient, due to the classical results of Birkhoff (1946) and of von Neumann (1953).

Theorem 1.1 For a bipartite graph $G:=(V, E)$, a vector $x$ in $\mathbb{R}^{E}$ belongs to $\mathcal{P}$ oly $(G)$ if and only if it is non-negative and 1-regular.

A graph $G:=(V, E)$ is Birkhoff-von Neumann if it satisfies the property that a vector $x$ in $\mathbb{R}^{E}$ belongs to $\operatorname{Poly}(G)$ if and only if it is non-negative and 1-regular. (Thus all bipartite graphs are Birkhoff-von Neumann.) The complete graph $K_{4}$ is also Birkhoff-von Neumann, whereas the triangular prism $\overline{C_{6}}$ is not. This suggests the following problem.

Problem 1.2 Characterize Birkhoff-von Neumann graphs. (Is the problem of deciding whether a given graph is Birkhoff-von Neumann in the complexity class $\mathcal{N} \mathcal{P}$ ? Is it in $\mathcal{P}$ ?)

\section{$1.2 \quad$ PM-compact graphs}

The combinatorial diameter of a polytope is the diameter of its 1-skeleton graph. A graph $G$ is $P M$-compact if the combinatorial diameter of $\mathcal{P}$ oly $(G)$ equals one, or equivalently, if the 1skeleton graph of $\mathcal{P}$ oly $(G)$ is a complete graph. Balinski and Russakoff [2], and independently Chvátal [7], showed that two vertices of the 1-skeleton graph of $\mathcal{P}$ oly $(G)$ are adjacent if and only if the symmetric difference of the corresponding perfect matchings is exactly one (even) cycle. Consequently, a graph $G$ is PM-compact if and only if the symmetric difference of any two perfect matchings of $G$ is exactly one cycle. For instance, each of $K_{4}$ and $\overline{C_{6}}$ is PM-compact, whereas the cube graph (on eight vertices) is not. This suggests the following problem.

Problem 1.3 Characterize PM-compact graphs. (Is the problem of deciding whether a given graph is $P M$-compact in the complexity class $\mathcal{N} \mathcal{P}$ ? Is it in $\mathcal{P}$ ?)

\subsection{Conformal bicycles}

A graph $G$ is matchable if it has a perfect matching. A connected graph $G$, of order at least two, is matching covered if each edge belongs to some perfect matching of $G$.

Using Edmond's algorithm, one may decide in polynomial-time whether or not an edge belongs to some perfect matching. Consequently, it is not difficult to see that the search for an answer to Problem 1.2, or for an answer to Problem 1.3, may be restricted to matching covered graphs. 
A subgraph $H$ of a matchable graph $G$ is conformal if $G-V(H)$ has a perfect matching. By a conformal bicycle, we mean a pair of vertex-disjoint cycles $\left(C_{1}, C_{2}\right)$ such that $G-V\left(C_{1}\right)-V\left(C_{2}\right)$ has a perfect matching. Since $|V(G)|$ is even, the parities of $C_{1}$ and of $C_{2}$ are the same. We say that the conformal bicycle $\left(C_{1}, C_{2}\right)$ is odd if each of $C_{1}$ and $C_{2}$ is an odd cycle; otherwise, it is even.

The aforementioned result of Balinski and Russakoff [2], and of Chvátal [7], implies the following characterization of PM-compact matchable graphs; see [15] for a proof.

Theorem 1.4 A matchable graph is PM-compact if and only if it does not contain an even conformal bicycle.

Consequently, every simple matchable graph on at most six vertices is PM-compact. However, this is not necessarily true when we allow multiple edges. For example, $K_{4}$ is PM-compact. However, $K_{4}$ with multiple edges may have two vertex-disjoint cycles (each of length two), in which case the graph is not PM-compact. On the other hand, if a graph $G$ is PM-compact then the underlying simple graph of $G$ is also PM-compact.

If a matchable graph $G$ has an odd conformal bicycle, then it is easy to construct a nonnegative 1-regular vector that does not belong to $\mathcal{P}$ oly $(G)$, whence $G$ is not Birkhoff-von Neumann. A result of Balas [1] shows that the converse holds as well.

Theorem 1.5 A matchable graph is Birkhoff-von Neumann if and only if it does not contain an odd conformal bicycle.

Consequently, every matchable graph (not necessarily simple) on at most four vertices is Birkhoff-von Neumann. Furthermore, a graph $G$ is Birkhoff-von Neumann if and only if the underlying simple graph of $G$ is Birkhoff-von Neumann.

It follows from Theorems 1.5 and 1.4, respectively, that the problems of deciding whether a graph $G$ is Birkhoff-von Neumann, and of deciding whether $G$ is PM-compact, both belong to the complexity class co- $\mathcal{N} \mathcal{P}$. However, to the best of our knowledge, it is not known whether either of these problems is in $\mathcal{N} \mathcal{P}$.

\subsection{Main result}

In this paper, we consider the "intersection" of these two problems. We provide an exact characterization of matching covered graphs that are Birkhoff-von Neumann as well as PM-compact. Consequently, the problem of deciding whether a graph is Birkhoff-von Neumann as well as PM-compact is in $\mathcal{P}$. Before stating our result precisely, we briefly mention special cases of Problems 1.2 and 1.3 that have already been solved in the literature.

The problem of characterizing Birkhoff--von Neumann graphs is equivalent to another important problem in matching theory — that of characterizing "solid" graphs; see [4]. Carvalho, Lucchesi and Murty [6] characterized the Birkhoff--von Neumann planar graphs. Wang, Lin, Carvalho, Lucchesi, Sanjith and Little [13] characterized PM-compact graphs 
that are bipartite or "near-bipartite", whereas Wang, Shang, Lin and Carvalho [14] characterized those that are cubic and claw-free.

Let $G$ be a matching covered graph and let $v$ be a vertex of degree two, with two distinct neighbours $u$ and $w$. The bicontraction of $v$ is the operation of contracting the two edges $v u$ and $v w$ incident with $v$. The retract of $G$ is the graph obtained from $G$ by bicontracting all its degree two vertices. It is easy to prove that the retract of a matching covered graph is also matching covered. Carvalho et al. [5] showed that the retract of a matching covered graph is unique up to isomorphism. The following facts are easily proved using the characterizations provided by Theorems 1.4 and [1.5, respectively.

Proposition 1.6 A matching covered graph is PM-compact if and only if its retract is PM-compact.

Proposition 1.7 A matching covered graph is Birkhoff-von Neumann if and only if its retract is Birkhoff-von Neumann.

Consequently, a matching covered graph $G$ is Birkhoff-von Neumann and PM-compact if and only if the retract of $G$ has each of these properties. Also, it is easily verified that, if $G$ is a matching covered graph of order at least four, and is not isomorphic to a simple cycle graph, then the retract of $G$ has minimum degree three or more. Thus, in order to characterize the matching covered graphs that are Birkhoff-von Neumann as well as PM-compact, we may restrict attention to matching covered graphs that have minimum degree three or more.

The following characterization of PM-compact bipartite matching covered graphs was obtained by Wang, Lin, Carvalho, Lucchesi, Sanjith and Little [13].

Theorem 1.8 The graphs $K_{2}$ (with multiple edges) and $K_{3,3}$ are the only PM-compact bipartite matching covered graphs that have minimum degree three or more.

The odd wheel $W_{2 k+1}$, for $k \geq 1$, is defined to be the join of an odd cycle $C_{2 k+1}$ and $K_{1}$. The smallest odd wheel is $K_{4}$. If $k \geq 2$, then $W_{2 k+1}$ has exactly one vertex of degree $2 k+1$, called its $h u b$, and the edges incident at the hub are called its spokes. The remaining $2 k+1$ vertices lie on a cycle, called the rim; vertices and edges of the rim are referred to as rim vertices and rim edges, respectively.

Each cycle of $W_{2 k+1}$ (possibly with multiple spokes), except for the rim, contains the hub; consequently this graph does not have two vertex-disjoint cycles, whence it is Birkhoff-von Neumann as well as PM-compact.

A vertex of a graph is cubic if its degree is precisely three; otherwise it is noncubic. A graph is cubic if all of its vertices are cubic.

We now describe two matching covered graphs that play an important role in our work. We obtain these graphs from $K_{3,3}$ with color classes $\left\{a_{1}, a_{2}, a_{3}\right\}$ and $\left\{b_{1}, b_{2}, b_{3}\right\}$. The first graph, denoted by $K_{4} \odot K_{3,3}$, is cubic, and is obtained from $K_{3,3}$ by replacing the vertex $a_{3}$ by a triangle, as shown in Figure1(a). This graph is a "splicing" of $K_{4}$ and $K_{3,3}$. The second 


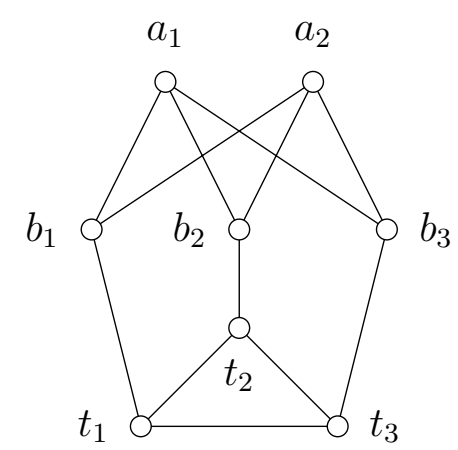

(a)

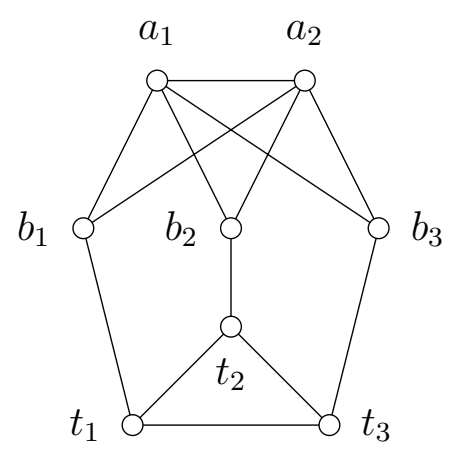

(b)

Figure 1: (a) $K_{4} \odot K_{3,3}$; (b) the Murty graph

graph, shown in Figure 1(b), is obtained from $K_{4} \odot K_{3,3}$ by adding an edge joining $a_{1}$ and $a_{2}$. This graph has precisely two noncubic vertices, and we shall refer to it as the Murty graph for reasons explained later. It is worth noting that, for both graphs shown in Figure 1, the automorphism group has precisely three orbits $-\left\{a_{1}, a_{2}\right\},\left\{b_{1}, b_{2}, b_{3}\right\}$ and $\left\{t_{1}, t_{2}, t_{3}\right\}$.

The reader may check that the Murty graph (possibly with multiple edges joining the two noncubic vertices) does not contain a conformal bicycle. Consequently, $K_{4} \odot K_{3,3}$ does not contain a conformal bicycle either. Our main result is the following.

Theorem 1.9 [The MAIN THEOREM] For a matching covered graph $G$, that has minimum degree three or more, the following are equivalent.

(i) G is Birkhoff-von Neumann as well as PM-compact.

(ii) $G$ does not contain a conformal bicycle.

(iii) $G$ is one of the following: (a) $K_{2}$ (with multiple edges), (b) $K_{3,3}$, (c) $K_{4}$ (up to multiple edges such that it does not have two vertex-disjoint cycles), (d) an odd wheel of order six or more (up to multiple spokes), (e) $K_{4} \odot K_{3,3}$, (f) the Murty graph (up to multiple edges joining the two noncubic vertices).

\section{Matching covered graphs}

For a cut $C:=\partial(X)$ of a graph $G$, we denote the graph obtained by contracting the shore $X$ to a single vertex $x$ by $G /(X \rightarrow x)$. In case the label of the contraction vertex $x$ is irrelevant, we simply write $G / X$. The graphs $G / X$ and $G / \bar{X}$ are called the $C$-contractions of $G$, and we say that $G$ is a splicing of these two graphs. 


\subsection{Tight cut decomposition}

Let $G$ be a matching covered graph. A cut $C$ is a tight cut if $|M \cap C|=1$ for every perfect matching $M$ of $G$. It is easily verified that if $C$ is a nontrivial tight cut of $G$, then each $C$-contraction is a matching covered graph that has strictly fewer vertices than $G$. If either of the $C$-contractions has a nontrivial tight cut, then that graph can be further decomposed into even smaller matching covered graphs. We can repeat this procedure until we obtain a list of matching covered graphs, each of which is free of nontrivial tight cuts. This procedure is known as a tight cut decomposition of $G$.

A matching covered graph free of nontrivial tight cuts is called a brace if it is bipartite; otherwise it is called a brick. Thus a tight cut decomposition of a matching covered graph results in a list of bricks and braces. For example, a tight cut decomposition of the graph $K_{4} \odot K_{3,3}$, shown in Figure 1(a), yields the brick $K_{4}$ and the brace $K_{3,3}$. Lovász [10] proved the following remarkable result.

Theorem 2.1 Any two tight cut decompositions of a matching covered graph yield the same list of bricks and braces (except possibly for multiplicities of edges).

In particular, any two tight cut decompositions of a matching covered graph $G$ yield the same number of bricks; this number is denoted by $b(G)$. We remark that $G$ is bipartite if and only if $b(G)=0$. We say that $G$ is a near-brick if $b(G)=1$. (For instance, $K_{4} \odot K_{3,3}$.) Thus, if $C$ is a nontrivial tight cut of a near-brick $G$, then one of its $C$-contractions is bipartite, whereas the other $C$-contraction is a near-brick (on fewer vertices).

It is worth noting that each vertex of a brick, or of a brace on at least six vertices, has at least three distinct neighbours.

\subsection{Solid graphs}

The tight cut decomposition can be performed in polynomial-time, whence the bricks and braces of a matching covered graph can be computed in polynomial-time. Carvalho, Lucchesi and Murty [4] proved the following — which implies that, in order to characterize Birkhoffvon Neumann graphs, it suffices to characterize Birkhoff--von Neumann bricks.

Theorem 2.2 A matching covered graph $G$ is Birkhoff-von Neumann if and only if either $G$ is bipartite or otherwise $G$ is a near-brick whose unique brick is Birkhoff-von Neumann.

We briefly discuss the class of "solid" matching covered graphs that plays a crucial role in several works of Carvalho, Lucchesi and Murty; see [3, 4]. As per the terminology we have already defined, a matching covered graph $G$ is solid if and only if each brick of $G$ (if any) is Birkhoff-von Neumann. Thus every Birkhoff-von Neumann matching covered graph is also solid. However, the converse is not true. In the case of bricks (as well as near-bricks), the two notions coincide exactly. Thus the problem of characterizing Birkhoff-von Neumann bricks is in fact the same as that of characterizing solid bricks. The following result of Carvalho, Lucchesi and Murty [6] implies that the problems of deciding whether a planar graph $G$ is Birkhoff-von Neumann, and of deciding whether $G$ is solid, are both in $\mathcal{P}$. 
Theorem 2.3 The odd wheels, up to multiple edges, are the only Birkhoff-von Neumann planar bricks.

A graph $G$ is odd-intercyclic if it does not contain two vertex-disjoint odd cycles. The odd wheels have this property. Clearly, every odd-intercyclic brick is Birkhoff-von Neumann. The Murty graph, shown in Figure 1(b), is the smallest brick that is Birkhoff-von Neumann, but is not odd-intercyclic. It is in this context that U. S. R. Murty first stumbled upon this graph (private communication), and it also appears in the work of Carvalho, Lucchesi and Murty [6, Figure 14].

\subsection{Proof of Theorem 1.9}

As is often the case in matching theory, it turns out that the most difficult part of proving the Main Theorem (1.9) is when the graph under consideration is a brick. In this section, we will assume the following characterization of bricks that are Birkhoff-von Neumann as well as PM-compact, and present a proof of Theorem 1.9 that relies on this assumption.

Theorem 2.4 A brick $G$ is Birkhoff-von Neumann as well as PM-compact if and only if $G$ is one of the following: (a) $K_{4}$ (up to multiple edges such that it does not have two vertexdisjoint cycles), (b) an odd wheel of order six or more (up to multiple spokes), (c) the Murty graph (up to multiple edges joining the two noncubic vertices).

A proof of the above result appears in Section [3. In order to prove Theorem 1.9, we will need a few more results. The following is easy to prove using the characterization of PM-compact graphs provided by Theorem 1.4. A proof appears in [13].

Proposition 2.5 Let $G$ be a PM-compact matching covered graph, and let $C$ be a nontrivial tight cut of $G$. Then each $C$-contraction of $G$ is PM-compact.

Likewise, the following is easy to prove using the characterization of Birkhoff-von Neumann graphs provided by Theorem 1.5 .

Proposition 2.6 Let $G$ be a Birkhoff-von Neumann matching covered graph, and let $C$ be a nontrivial tight cut of $G$. Then each $C$-contraction of $G$ is Birkhoff-von Neumann.

Finally, we will need the following lemma in order to prove Theorem 1.9 using induction.

Lemma 2.7 Let $G$ be a matching covered graph that has minimum degree three or more. If $G$ has a nontrivial tight cut, then $G$ has a nontrivial tight cut that has at least three edges.

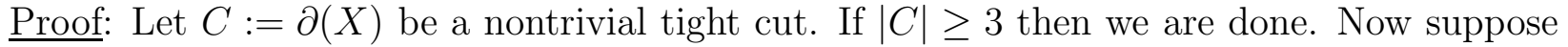
that $|C|=2$, and let $C:=\{u \bar{u}, v \bar{v}\}$ such that $u, v \in X$ and $\bar{u}, \bar{v} \in \bar{X}$. We let $Y:=X-u+\bar{v}$ and $D:=\partial(Y)$. Clearly, $|Y|=|X|$; consequently, $D$ is a nontrivial cut and both of its shores are of odd cardinality. Observe that each edge of $D$ is incident either with $u$ or with $\bar{v}$. Thus 
each perfect matching meets $D$ in precisely one edge; whence $D$ is a (nontrivial) tight cut. Finally, we infer that $|D| \geq 4$ since $G$ has minimum degree three or more. This completes the proof of Lemma 2.7 .

We are now ready to prove the Main Theorem (1.9) — under the assumption that Theorem 2.4 holds.

Proof of Theorem 1.9: The equivalence of the first two statements follows from Theorems 1.4 and 1.5. As discussed earlier, the graphs mentioned in statement (iii) do not have a conformal bicycle, whence (iii) implies (ii). Our task is to prove that (ii) implies (iii).

Suppose that $G$ is a matching covered graph that has minimum degree three or more and that does not have a conformal bicycle. We will show that $G$ is one of the graphs mentioned in statement (iii). We proceed by induction on the number of edges.

If $G$ is bipartite, or if $G$ is a brick, then we are done by Theorem 1.8, or by Theorem 2.4, respectively. Now suppose that $G$ is nonbipartite, and that it has a nontrivial tight cut. Lemma 2.7 implies that $G$ has a nontrivial tight cut $C:=\partial(X)$ such that $|C| \geq 3$. We let $G_{1}:=G / \bar{X} \rightarrow \bar{x}$ and $G_{2}:=G / X \rightarrow x$ denote the two $C$-contractions of $G$. Each of them is a matching covered graph with fewer edges. Since $|C| \geq 3$, each of $G_{1}$ and $G_{2}$ has minimum degree three or more. Also, Propositions 2.6 and 2.5 imply that each of $G_{1}$ and $G_{2}$ is Birkhoff-von Neumann as well as PM-compact. Theorem 2.2 implies that $G$ is a near-brick. Consequently, exactly one of $G_{1}$ and $G_{2}$ is bipartite; adjust notation so that $G_{2}$ is bipartite. It follows from the induction hypothesis that $G_{2}$ is $K_{3,3}$. In particular, each vertex of $G_{2}$, including the contraction vertex $x$, is cubic; whence $|C|=3$ and the contraction vertex $\bar{x}$ of $G_{1}$ is also cubic. We label the vertices of $G_{2}$ so that its color classes are $\left\{a_{1}, a_{2}, x\right\}$ and $\left\{b_{1}, b_{2}, b_{3}\right\}$. By the induction hypothesis, we have four possibilities for the graph $G_{1}$, and we consider each of them separately.

Case 1: $G_{1}$ is $K_{4}$ (up to multiple edges).

Note that the underlying simple graph of $G$ is $K_{4} \odot K_{3,3}$. If $G$ is simple then there is nothing to prove. Otherwise $G$ has a spanning subgraph $H$ that is isomorphic to the graph shown in Figure 2 (left). The reader may verify that $H$ has an even conformal bicycle, whence so does $G$, contrary to our assumption.

Case 2: $G_{1}$ is an odd wheel of order six or more (up to multiple spokes).

Since the contraction vertex $\bar{x}$ of $G_{1}$ is cubic, it lies on the rim of $G_{1}$. We let $h$ denote the hub of $G_{1}$, and we label the remaining vertices of $G_{1}$ so that $G_{1}-h$ is the cycle $\left(\bar{x}, w_{1}, w_{2}, \ldots w_{2 k}, \bar{x}\right)$. Note that $k \geq 2$. Adjust notation so that the cut $C=\left\{b_{1} w_{1}, b_{2} h, b_{3} w_{2 k}\right\}$. Figure 2 (right) shows the underlying simple graph when $k=2$. Now let $C_{1}$ denote the even cycle $\left(w_{1}, w_{2}, \ldots, w_{2 k-1}, h, w_{1}\right)$, and let $C_{2}$ denote the 4-cycle $\left(a_{1}, b_{1}, a_{2}, b_{2}, a_{1}\right)$. Observe that $\left(C_{1}, C_{2}\right)$ is an even conformal bicycle of $G$, contrary to our assumption.

Case 3: $G_{1}$ is $K_{4} \odot K_{3,3}$.

The graph $G$ is a splicing of two cubic graphs, namely $G_{1}=K_{4} \odot K_{3,3}$ and $G_{2}=K_{3,3}$. It follows from the automorphisms of $G_{1}$ that $G$ is isomorphic to one of three graphs shown in 

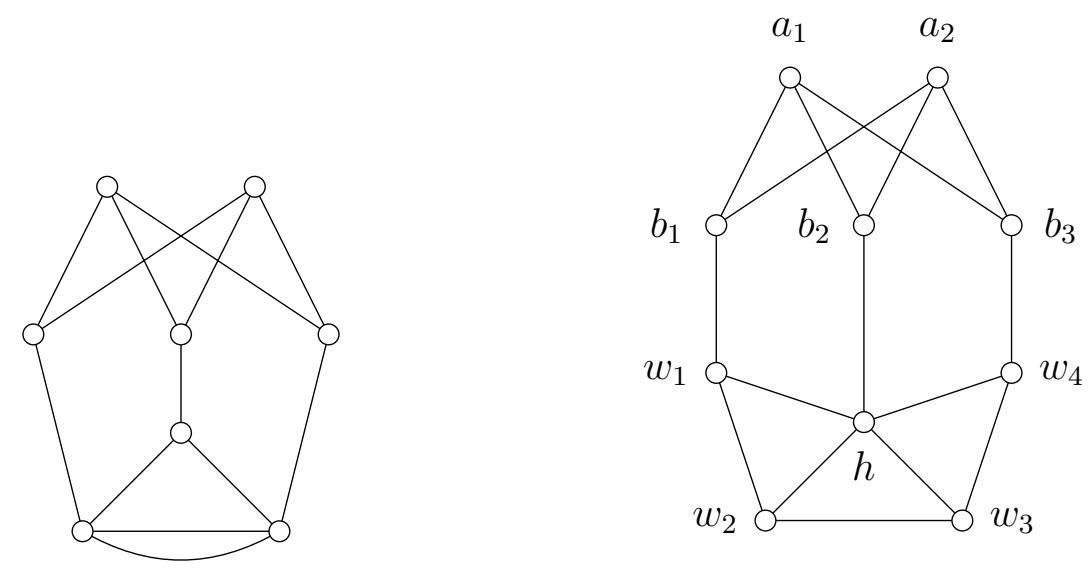

Figure 2: Illustrations for cases 1 and 2 in the proof of Theorem 1.9

Figure 3. Each of these three graphs has an even conformal bicycle that is shown using bold lines; this contradicts our assumption.

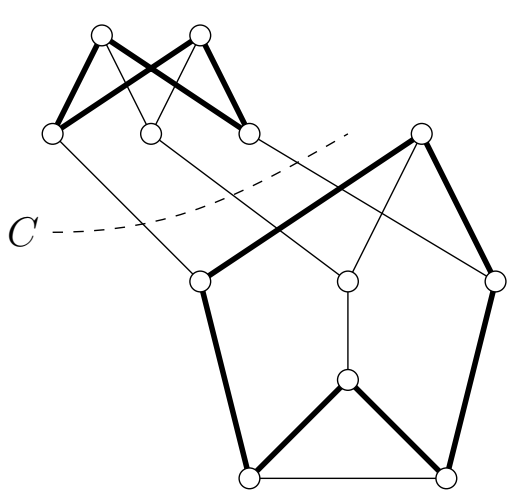

(a)

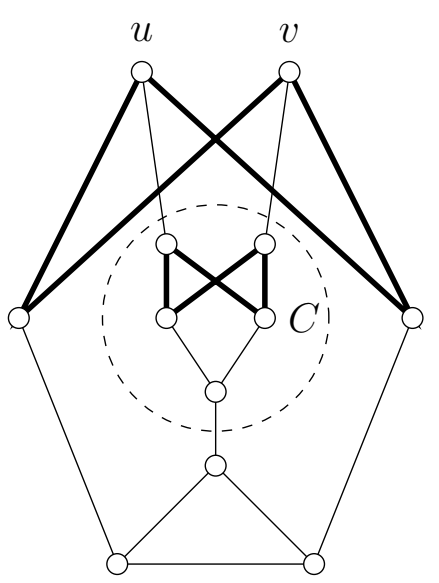

(b)

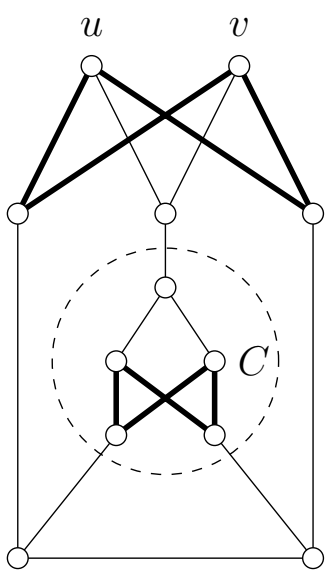

(c)

Figure 3: Illustrations for cases 3 and 4 in the proof of Theorem 1.9

Case 4: $G_{1}$ is the Murty graph (up to multiple edges joining the two noncubic vertices). The graph $G$ is a splicing of the Murty graph (that is, $G_{1}$ ) and $G_{2}=K_{3,3}$. Let $u$ and $v$ denote the two noncubic vertices of $G_{1}$. Since the contraction vertex $\bar{x}$ of $G_{1}$ is cubic, it is distinct from $u$ and $v$. It follows from the automorphisms of $G_{1}$ that the underlying simple graph of $G$ is isomorphic to either the graph shown in Figure $3(b)$ plus the edge $u v$, or to the graph shown in Figure 3(c) plus the edge $u v$. In either case, $G$ contains an even conformal bicycle, contrary to our assumption.

This completes the proof of the Main Theorem (1.9). 


\section{Bricks}

Recall that bricks are those nonbipartite matching covered graphs that are free of nontrivial tight cuts. Edmonds, Lovász and Pulleyblank [8] proved the following deep result.

Theorem 3.1 A graph $G$, of order at least four, is a brick if and only if $G-u-v$ is connected and matchable, for all pairs of distinct vertices $u, v \in V(G)$.

A consequence worth noting is that adding an edge to a brick yields another brick. In this section, our goal is to prove Theorem 2.4 that provides a complete characterization of bricks that are Birkhoff-von Neumann and PM-compact. However, before that, we discuss induction tools from existing literature that are useful in proving results concerning bricks.

\subsection{Strictly thin edges}

An edge $e$ of a brick $G$ is thin if the retract of $G-e$ is also a brick. Carvalho, Lucchesi and Murty [6] proved the following result.

Theorem 3.2 Every brick, distinct from $K_{4}, \overline{C_{6}}$ and the Petersen graph, has a thin edge.

Carvalho et al. 6] also described four simple expansion operations that may be applied to any brick to obtain a larger brick. Given a brick $H$, the application of any of those four operations to $H$ results in a brick $G$ such that $G$ has a thin edge $e$ with the property that $H$ is the retract of $G-e$. We refer the reader to their work for a precise description of the expansion operations. Their importance is due to the following result; see [6, Theorem 36].

Theorem 3.3 Let $H$ be a brick and let $G$ be a graph obtained from $H$ by one of the four expansion operations. Then $G$ is a brick.

Thus, any brick may be generated from one of the three bricks $\left(K_{4}, \overline{C_{6}}\right.$ and the Petersen graph) by means of the four expansion operations. In the same paper, Carvalho et al. used this generation procedure to prove Theorem 2.3 that provides a complete characterization of Birkhoff-von Neumann planar bricks.

However, one of the problems with this generation procedure is that in order to generate certain simple bricks 1 (such as the odd wheels of order six or more) one may have to allow intermediate bricks that are not necessarily simple. When proving other results using this generation procedure, the presence of multiple edges requires greater case analyis. In order to circumvent this difficulty, we shall instead use the stronger notion of a strictly thin edge, as is done in the work of Kothari and Murty [9].

A thin edge $e$ of a simple brick $G$ is strictly thin if the retract of $G-e$ is a simple brick. With each strictly thin edge $e$ of $G$ there is an associated number, called its index, which is:

\footnotetext{
${ }^{1} \mathrm{~A}$ simple brick is a brick that is devoid of multiple edges.
} 
- zero, if both ends of $e$ are noncubic in $G$;

- one, if exactly one end of $e$ is cubic in $G$;

- two, if both ends of $e$ are cubic in $G$, and $e$ does not lie in a triangle;

- three, if both ends of $e$ are cubic in $G$, and $e$ lies in a triangle.

The following proposition is easily verified (and appears in [9]).

Proposition 3.4 Let $G$ be a simple brick, and let e denote a strictly thin edge of $G$, and let $H$ be the retract of $G-e$. If the index of $e$ is zero, then $H=G-e$. If the index of $e$ is one, then $G-e$ has precisely one vertex of degree two; and $H$ has just one contraction vertex, and its degree is at least four. If the index of e is two, then $G-e$ has precisely two vertices of degree two, and they have no common neighbour; and $H$ has two contraction vertices, and their degrees are at least four. If the index of e is three, then $G-e$ has precisely two vertices of degree two, and they have a common neighbour; and $H$ has just one contraction vertex, and its degree is at least five.

We refer the reader to [9, Figure 2] for examples of strictly thin edges. It is easily verified that odd wheels do not have strictly thin edges. There are four other infinite families of simple bricks that are devoid of strictly thin edges. These are prisms, truncated biwheels, staircases and Möbius ladders. We refer the reader to [9] for a description of these families. Norine and Thomas [12] proved the following.

Theorem 3.5 Let $G$ be a simple brick. If $G$ has no strictly thin edge then $G$ is either the Petersen graph or is an odd wheel, a prism, a truncated biwheel, a staircase or a Möbius ladder.

As is done in [9], we say that a simple brick is Norine-Thomas if it is free of strictly thin edges. (Thus, these are exactly the graphs that appear in the above theorem statement.) The following is an immediate consequence of Theorem 3.5 .

Theorem 3.6 Given any simple brick $G$, there exists a sequence $G_{1}, G_{2}, \ldots, G_{r}$ of simple bricks such that (i) $G_{1}$ is a Norine-Thomas brick; (ii) $G_{r}=G$; and (iii) for $2 \leq i \leq r, G_{i}$ has a strictly thin edge $e_{i}$ such that $G_{i-1}$ is the retract of $G_{i}-e_{i}$.

The above theorem implies that every simple brick can be generated from one of the Norine-Thomas bricks by repeated application of the expansion operations in some sequence, such that at each step we have a simple brick. Kothari and Murty [9] used this generation procedure to provide complete characterizations of planar bricks that are $K_{4}$-free, and those that are $\overline{C_{6}}$-free.

We will use this generation procedure in order to prove Theorem 2.4, Let $G$ be a brick and let $e$ be a thin edge of $G$. If $G$ is PM-compact, then it follows from Theorem 1.4 that $G-e$ is also PM-compact, and so is the retract of $G-e$ by invoking Proposition [1.6. A similar fact holds for the Birkhoff-von Neumann property by invoking Theorem 1.5 and Proposition 1.7. 
Proposition 3.7 Let $G$ be a brick, let e be a thin edge of $G$, and let $H$ be the retract of $G-e$. Then the following statements hold.

(i) If $G$ is Birkhoff-von Neumann then $H$ is also Birkhoff-von Neumann.

(ii) If $G$ is $P M$-compact then $H$ is also PM-compact.

In order to characterize the bricks that are Birkhoff-von Neumann as well as PM-compact, we first need to find out which Norine-Thomas bricks have both of these properties. This is done easily using Theorems 1.4 and 1.5 .

Proposition 3.8 The odd wheels are the only Norine-Thomas bricks that are Birkhoff-von Neumann as well as PM-compact.

Proof: Let $G$ be any Norine-Thomas brick. If $G$ is a prism, or a truncated biwheel, or a staircase, or the Petersen graph, then $G$ has an odd conformal bicycle, whence $G$ is not Birkhoff-von Neumann. On the other hand, if $G$ is a Möbius ladder of order eight or more, then $G$ has an even conformal bicycle. (The only other Möbius ladder is $K_{4}$ which is also an odd wheel.) Consequently, if $G$ is Birkhoff-von Neumann and PM-compact, then $G$ is indeed an odd wheel.

It follows from Propositions 3.7 and 3.8 that, if $G$ is a simple brick that is Birkhoffvon Neumann and PM-compact, and if $G_{1}, G_{2}, \ldots, G_{r}$ is a sequence of simple bricks as in Theorem 3.6, then each $G_{i}$ is also Birkhoff-von Neumann and PM-compact, and furthermore, $G_{1}$ is in fact an odd wheel.

In order to prove Theorem 2.4, we need to investigate how "new" bricks that are Birkhoffvon Neumann as well as PM-compact may be generated from the odd wheels, and also from the Murty graph (which is in turn generated from $W_{5}$ as we will see soon). The next four sections are devoted to this task. Most of the proofs are straightforward. We will make extensive use of Theorems 1.4 and 1.5 without referring to them explicitly.

\subsection{Adding an edge}

In this section, we consider the simplest operation - that of adding an edge.

Proposition 3.9 Let $G$ be a graph obtained from $W_{2 k+1}$, where $k \geq 2$, by adding an edge $e$ joining any two rim vertices. Then either $G$ is not Birkhoff-von Neumann, or $G$ is not PM-compact, possibly both.

Proof: We let $u$ and $v$ denote the ends of $e$, and let $h$ denote the hub of $W_{2 k+1}$. If $u$ and $v$ are adjacent in $W_{2 k+1}$, then there is a 2-cycle $C_{1}$ containing $u$ and $v$, and there is a cycle containing all of the remaining $2 k$ vertices, whence $\left(C_{1}, C_{2}\right)$ is an even conformal bicycle and $G$ is not PM-compact. Now suppose that $u$ and $v$ are nonadjacent in $W_{2 k+1}$. The rim of $W_{2 k+1}$ is a union of two internally-disjoint $u v$-paths, say $P_{1}$ and $P_{2}$. One of them, say $P_{1}$, 
is of odd length, and has length at least three. We let $Q_{1}:=P_{1}-u-v$, and let $u^{\prime}$ and $v^{\prime}$ denote the ends of $Q_{1}$. Let $C_{1}:=Q_{1}+v^{\prime} h+h u^{\prime}$ and $C_{2}:=P_{2}+e$. Observe that $\left(C_{1}, C_{2}\right)$ is an odd conformal bicycle, whence $G$ is not Birkhoff-von Neumann.

Proposition 3.10 Let $H$ denote the Murty graph, and let $a_{1}$ and $a_{2}$ denote its noncubic vertices. Let $G$ be a graph obtained from $H$ by adding an edge e such that at least one of $a_{1}$ and $a_{2}$ is not an end of e. Then either $G$ is not Birkhoff-von Neumann, or $G$ is not PM-compact, possibly both.

Proof: We label the graph $H$ as in Figure 1(b), and we let $u$ and $v$ denote the ends of $e$. First suppose that $u$ and $v$ are adjacent in $H$. Up to symmetry, there are three cases: $\{u, v\}=\left\{t_{1}, t_{3}\right\},\{u, v\}=\left\{t_{1}, b_{1}\right\}$ and $\{u, v\}=\left\{a_{1}, b_{1}\right\}$. In each case, $G$ has an even conformal bicycle. Now suppose that $u$ and $v$ are nonadjacent in $H$. Up to symmetry, there are three cases: $\{u, v\}=\left\{b_{1}, b_{2}\right\},\{u, v\}=\left\{t_{1}, b_{2}\right\}$ and $\{u, v\}=\left\{t_{1}, a_{1}\right\}$. In each case, $G$ has either an even conformal bicycle, or an odd conformal bicycle. (We omit the details.)

In the next three sections, we consider the remaining three expansion operations. However, for convenience, we shall instead think of deleting a strictly thin edge $e$ from a brick $G$ and taking the retract in order to obtain a smaller brick $H$ that is either an odd wheel or is the Murty graph. We will invoke Proposition 3.4 without referring to it explicitly. We shall adopt the following notation and conventions.

Notation 3.11 For a strictly thin edge e of a simple brick $G$, we let $e:=u_{0} v_{0}$, and we let $H$ denote the retract of $G-e$. If $u_{0}$ is cubic in $G$, we let $u_{1}$ and $u_{2}$ denote the neighbours of $u_{0}$ that are distinct from $v_{0}$. Likewise, if $v_{0}$ is cubic in $G$, we let $v_{1}$ and $v_{2}$ denote the neighbours of $v_{0}$ that are distinct from $u_{0}$. If the index of e is one then we adjust notation so that $u_{0}$ is cubic and $v_{0}$ is noncubic. If the index of $e$ is three then $u_{0}$ and $v_{0}$ have a common neighbour, and we adjust notation so that $u_{1}=v_{1}$.

\subsection{Index one}

Proposition 3.12 Let $G$ be a simple brick, and let e be a strictly thin edge of index one such that the retract of $G-e$ is the Murty graph. Then either $G$ is not Birkhoff-von Neumann, or $G$ is not PM-compact, possibly both.

Proof: We adopt Notation 3.11, We label the vertices of $H$ as shown in Figure 1(b). The contraction vertex of $H$ resulting from the bicontraction of $u_{0}$ has degree at least four, whence one of $a_{1}$ and $a_{2}$ is the contraction vertex. Adjust notation so that $a_{1}$ is the contraction vertex. The graph $H-a_{1}$ is shown in Figure 4. In $G$, precisely two vertices from the set $\left\{a_{2}, b_{1}, b_{2}, b_{3}\right\}$ are neighbours of $u_{1}$, and the remaining two are neighbours of $u_{2}$. By observing the symmetries of $H-a_{1}$, we may adjust notation so that $b_{1}$ and $b_{2}$ are neighbours of $u_{1}$, whereas $b_{3}$ and $a_{2}$ are neighbours of $u_{2}$, as shown in Figure 4 . 

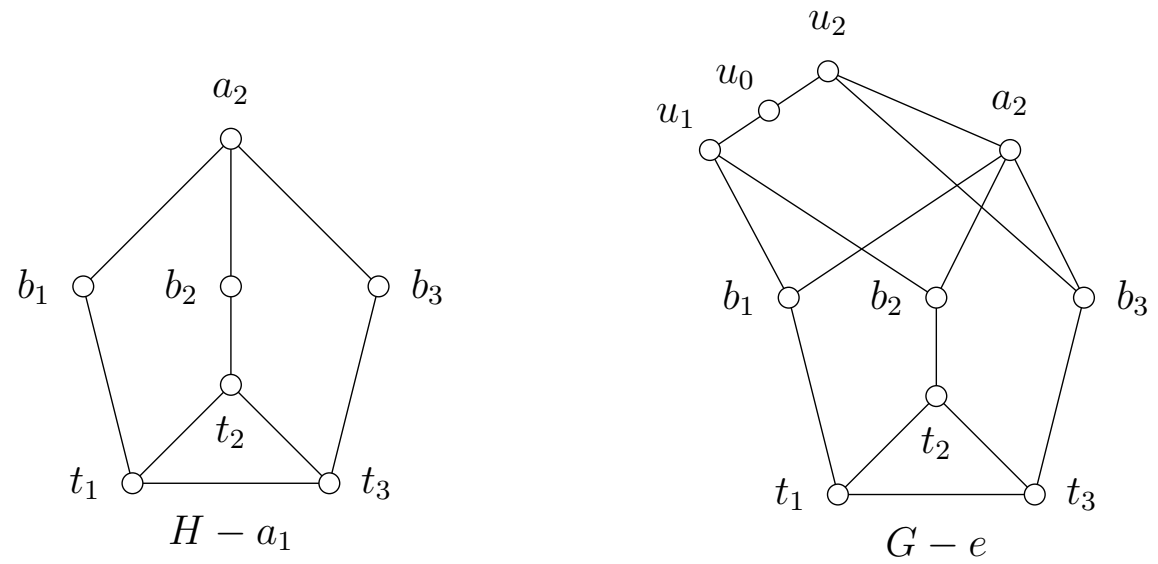

Figure 4: Illustration for the proof of Proposition 3.12

In $G-e$, there is an automorphism that swaps $t_{1}$ and $t_{2}$, as well as $b_{1}$ and $b_{2}$, and keeps remaining vertices fixed. Thus, up to symmetry, there are five possibilities for the end $v_{0}$ of the edge $e$. These are: $v_{0} \in\left\{t_{1}, b_{1}, t_{3}, b_{3}, a_{2}\right\}$. In each case, we present a conformal bicycle $\left(C_{1}, C_{2}\right)$. If $v_{0}=t_{1}$ then let $C_{1}:=\left(t_{1}, u_{0}, u_{1}, b_{1}, t_{1}\right)$ and $C_{2}:=\left(b_{3}, u_{2}, a_{2}, b_{2}, t_{2}, t_{3}, b_{3}\right)$. If $v_{0}=b_{1}$ then let $C_{1}:=\left(b_{1}, u_{0}, u_{1}, b_{1}\right)$ and $C_{2}:=\left(u_{2}, a_{2}, b_{3}, u_{2}\right)$. If $v_{0}=t_{3}$ then let $C_{1}:=\left(t_{3}, u_{0}, u_{1}, b_{1}, t_{1}, t_{3}\right)$ and $C_{2}:=\left(u_{2}, a_{2}, b_{3}, u_{2}\right)$. If $v_{0}=b_{3}$ then let $C_{1}:=\left(b_{3}, u_{0}, u_{2}, b_{3}\right)$ and $C_{2}:=\left(t_{1}, t_{2}, t_{3}, t_{1}\right)$. If $v_{0}=a_{2}$ then let $C_{1}:=\left(a_{2}, u_{0}, u_{2}, a_{2}\right)$ and $C_{2}:=\left(t_{1}, b_{1}, u_{1}, b_{2}, t_{2}, t_{1}\right)$. In all cases, we conclude that either $G$ is not Birkhoff-von Neumann, or $G$ is not PM-compact, possibly both. This completes the proof of Proposition 3.12.

Proposition 3.13 Let $G$ be a simple brick, and let e be a strictly thin edge of index one such that the retract of $G-e$ is the odd wheel $W_{5}$. Then exactly one of the following holds:

(i) $G$ is the Murty graph.

(ii) Either $G$ is not Birkhoff-von Neumann, or $G$ is not PM-compact, possibly both.

Proof: We adopt Notation 3.11. The contraction vertex of $H$ resulting from the bicontraction of $u_{0}$ has degree at least four, whence the hub of $H$ is indeed the contraction vertex. We label the rim of $H$, in cyclic order, as follows: $\left(w_{0}, w_{1}, w_{2}, w_{3}, w_{4}, w_{0}\right)$. Since the hub is incident with each vertex on the rim, we infer that one of $u_{1}$ and $u_{2}$ is incident with exactly two rim vertices, and the other is incident with the remaining three rim vertices. Adjust notation so that $u_{1}$ is incident with two rim vertices; there are two cases depending on whether these neighbours of $u_{1}$ appear consecutively on the rim (see Figure 5a), or not (see Figure 5b).

First suppose that the two neighbours of $u_{1}$ on the rim appear consecutively, and adjust notation so that $u_{1} w_{2}, u_{1} w_{3} \in E(G)$. Thus $u_{2} w_{4}, u_{2} w_{0}, u_{2} w_{1} \in E(G)$. Now, up to symmetry, there are three possibilities for the end $v_{0}$ of the edge $e$. These are $v_{0} \in\left\{w_{0}, w_{1}, w_{2}\right\}$. If $v_{0}=w_{0}$ then it is easily verified that $G$ is the Murty graph. In the remaining two cases, we 


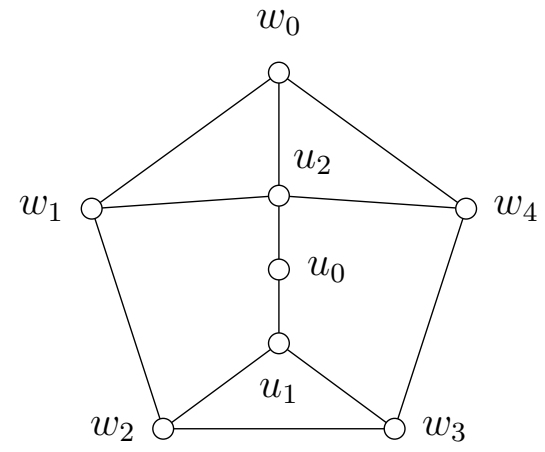

(a)

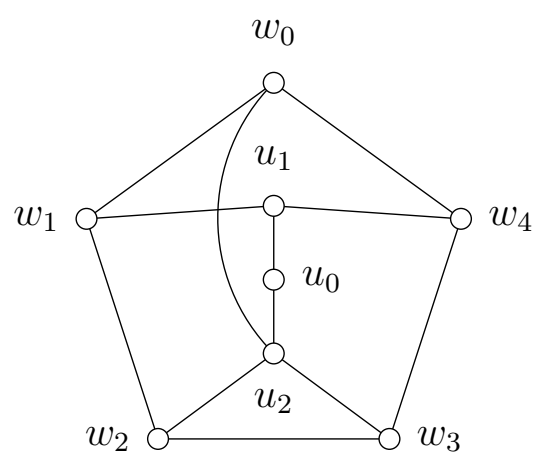

(b)

Figure 5: Illustration for the proof of Proposition 3.13

present an odd conformal bicycle $\left(C_{1}, C_{2}\right)$. If $v_{0}=w_{1}$ then let $C_{1}:=\left(w_{1}, u_{0}, u_{2}, w_{1}\right)$ and let $C_{2}:=\left(w_{2}, u_{1}, w_{3}, w_{2}\right)$. If $v_{0}=w_{2}$ then let $C_{1}:=\left(w_{2}, u_{0}, u_{1}, w_{2}\right)$ and let $C_{2}:=\left(w_{0}, u_{2}, w_{1}, w_{0}\right)$.

Now suppose that the two neighbours of $u_{1}$ on the rim do not appear consecutively, and adjust notation so that $u_{1} w_{1}, u_{1} w_{4} \in E(G)$. Thus $u_{2} w_{0}, u_{2} w_{2}, u_{2} w_{3} \in E(G)$. Now, up to symmetry, there are three possibilities for the end $v_{0}$ of the edge $e$. These are $v_{0} \in\left\{w_{0}, w_{1}, w_{2}\right\}$. In each case, we present a conformal bicycle $\left(C_{1}, C_{2}\right)$. If $v_{0}=w_{0}$ then let $C_{1}:=\left(w_{0}, u_{0}, u_{2}, w_{0}\right)$ and let $C_{2}:=\left(u_{1}, w_{1}, w_{2}, w_{3}, w_{4}, u_{1}\right)$. If $v_{0}=w_{1}$ then let $C_{1}:=$ $\left(w_{1}, u_{0}, u_{1}, w_{1}\right)$ and let $C_{2}:=\left(w_{2}, u_{2}, w_{3}, w_{2}\right)$. If $v_{0}=w_{2}$ then let $C_{1}:=\left(w_{2}, u_{0}, u_{2}, w_{3}, w_{2}\right)$ and let $C_{2}:=\left(u_{1}, w_{4}, w_{0}, w_{1}, u_{1}\right)$.

This completes the proof of Proposition 3.13.

Lemma 3.14 Let $G$ be a simple brick, and let e be a strictly thin edge of index one such that the retract of $G-e$ is an odd wheel $W_{2 k+1}$ for some $k \geq 3$. Then $G$ has distinct edges $f_{1}$ and $f_{2}$, each of which is distinct from e, such that the following hold:

(i) $f_{1}$ is strictly thin of index one in $G$; let $H_{1}$ denote the retract of $G-f_{1}$;

(ii) $f_{2}$ is strictly thin of index zero in $H_{1}$; let $H_{2}:=H_{1}-f_{2}$;

(iii) $e$ is strictly thin of index one in $\mathrm{H}_{2}$, and the retract of $\mathrm{H}_{2}-e$ is the odd wheel $W_{2 k-1}$.

Proof: We adopt Notation 3.11, Adjust notation so that $\left|\partial\left(u_{1}\right)\right| \geq\left|\partial\left(u_{2}\right)\right|$. The contraction vertex of $H$ resulting from the bicontraction of $u_{0}$ has degree at least four, whence the hub of $H$ is indeed the contraction vertex. We let $Q$ denote the $\operatorname{rim}$ of $H$. Note that $Q$ is a cycle of length $2 k+1$ in $G$, and each vertex of $Q$ is a neighbour of exactly one of $u_{1}$ and $u_{2}$. Since $2 k+1 \geq 7$, the vertex $u_{1}$ has at least four neighbours in $V(Q)$, consequently $\left|\partial\left(u_{1}\right)\right| \geq 5$. Furthermore, since $\left|\partial\left(u_{1}\right)\right| \geq\left|\partial\left(u_{2}\right)\right|$, the cycle $Q$ has two consecutive vertices such that each of them is a neighbour of $u_{1}$. 


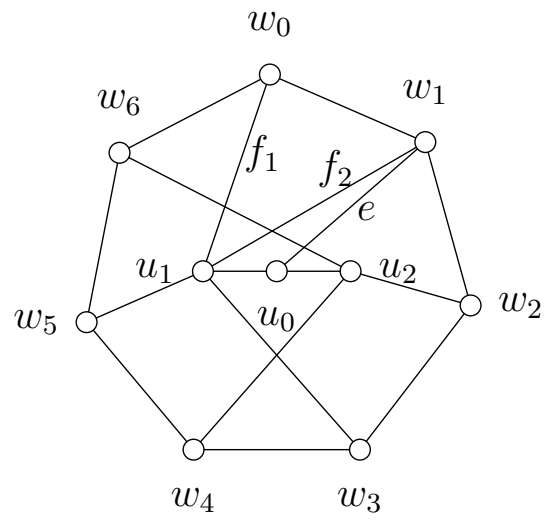

Figure 6: Illustration for the proof of Lemma 3.14

We choose a maximal path $P$, in $Q$, such that $|V(P)| \geq 2$ and each vertex of $P$ is a neighbour of $u_{1}$. Since $u_{2}$ has at least two neighbours in $V(Q),|V(P)| \leq 2 k-1$. At least one of the ends of $P$ is distinct from the end $v_{0}$ of edge $e$. We label the vertices of $Q$, in cyclic order: $\left(w_{0}, w_{1}, \ldots, w_{2 k}, w_{0}\right)$, such that $P:=\left(w_{0}, w_{1}, \ldots, w_{i}\right)$ and $w_{0} \neq v_{0}$. Note that $w_{1} u_{1}, w_{2 k} u_{2} \in E(G)$. We let $f_{1}:=w_{0} u_{1}$ and $f_{2}:=w_{1} u_{1}$. See Figure 6 for an example.

Observe that only one end of $f_{1}$, namely $w_{0}$, is cubic. We let $H_{1}$ denote the retract of $G-f_{1}$. Note that $H_{1}$ is simple, and that its unique contraction vertex is incident with the edge $f_{2}$. Consequently, each end of $f_{2}$ is noncubic in $H_{1}$. We let $H_{2}:=H_{1}-f_{2}$. Observe that exactly one end of $e$, namely $u_{0}$, is cubic in $H_{2}$, and that the retract of $H_{2}-e$ is indeed the odd wheel $W_{2 k-1}$. By Theorem $3.3, H_{2}$ is a (simple) brick, and $e$ a strictly thin edge of index one in $H_{2}$. Since $H_{1}$ is obtained from $H_{2}$ by adding an edge, $H_{1}$ is also a (simple) brick and $f_{2}$ a strictly thin edge of index zero in $H_{1}$. Consequently, $f_{1}$ is a strictly thin edge of index one in $G$. This completes the proof of Lemma 3.14.

We now have the following consequence by repeatedly applying Lemma 3.14.

Corollary 3.15 Let $G$ be a simple brick, and let e be a strictly thin edge of index one such that the retract of $G-e$ is an odd wheel. Then there exists a sequence $G_{1}, G_{2}, \ldots, G_{r}$ of simple bricks such that (i) $G_{1}=W_{5}$; (ii) $G_{r}=G$; (iii) for $2 \leq i \leq r, G_{i}$ has a strictly thin edge $e_{i}$ such that $G_{i-1}$ is the retract of $G_{i}-e_{i}$; and (iv) $e_{2}$ is of index one in $G_{2}$; and (v) if $r \geq 3$ then $e_{3}$ is of index zero in $G_{3}$.

\subsection{Index two}

Proposition 3.16 Let $G$ be a simple brick, and let e be a strictly thin edge of index two such that the retract of $G-e$ is the Murty graph. Then $G$ is neither Birkhoff-von Neumann nor PM-compact. 
Proof: We adopt Notation 3.11. We label the vertices of $H$ as shown in Figure 17. Each of the contraction vertices of $H$, resulting from the bicontraction of $u_{0}$ and of $v_{0}$, has degree at least four. Thus $a_{1}$ and $a_{2}$ are indeed the contraction vertices. Since $a_{1} a_{2} \in E(H)$, we infer that $G$ has exactly one edge joining a vertex in $\left\{u_{1}, u_{2}\right\}$ with a vertex in $\left\{v_{1}, v_{2}\right\}$. Adjust notation so that $u_{2} v_{2}$ is an edge of $G$.

The vertex $v_{1}$ has precisely two neighbours in the set $\left\{b_{1}, b_{2}, b_{3}\right\}$, and we may adjust notation so that $v_{1} b_{2}, v_{1} b_{3} \in E(G)$. Now, since $H$ is simple, we infer that $v_{2}$ is not adjacent with either of $b_{2}$ and $b_{3}$. Consequently, $v_{2} b_{1} \in E(G)$. Furthermore, $u_{1}$ is adjacent with at least one of $b_{2}$ and $b_{3}$, and we may adjust notation so that $u_{1} b_{3} \in E(G)$. Thus the graph shown in Figure 7 (right) is a subgraph of $G$.
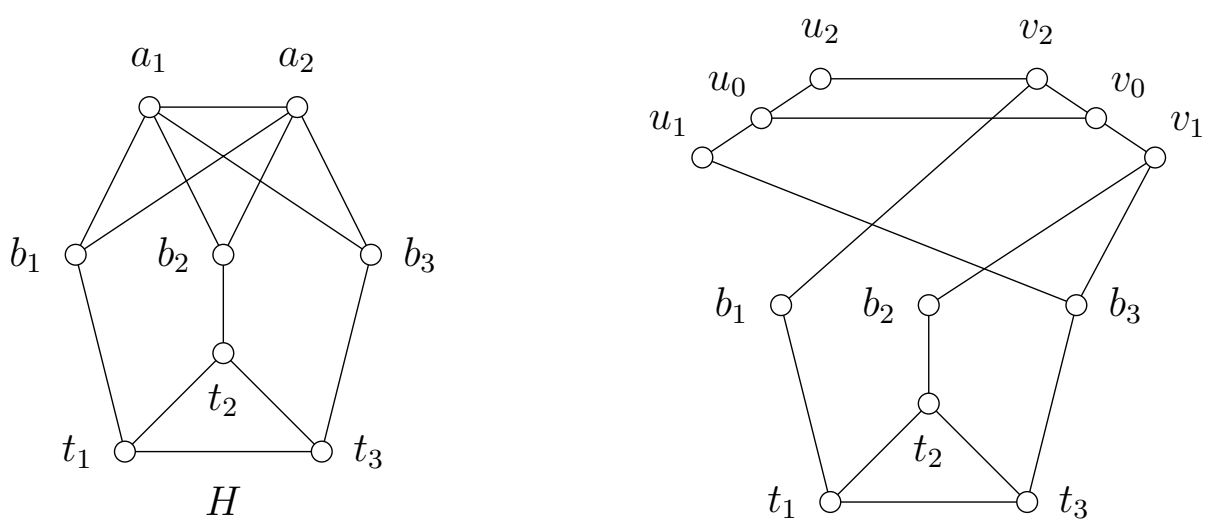

Figure 7: Illustration for the proof of Proposition 3.16

Now there are two possibilities: either $u_{1} b_{1}, u_{2} b_{2} \in E(G)$, or otherwise $u_{1} b_{2}, u_{2} b_{1} \in$ $E(G)$. In each case, we present an even conformal bicycle $\left(C_{1}, C_{2}\right)$, and we leave it to the reader to find an odd conformal bicycle. Let $C_{1}:=\left(u_{2}, v_{2}, v_{0}, u_{0}, u_{2}\right)$. If $u_{1} b_{1}, u_{2} b_{2} \in E(G)$ then let $C_{2}:=\left(t_{1}, t_{2}, b_{2}, v_{1}, b_{3}, t_{3}, t_{1}\right)$. If $u_{1} b_{2}, u_{2} b_{1} \in E(G)$ then let $C_{2}:=\left(u_{1}, b_{2}, v_{1}, b_{3}, u_{1}\right)$. Thus $G$ is neither Birkhoff-von Neumann nor PM-compact. This completes the proof of Proposition 3.16.

\subsection{Index three}

Proposition 3.17 Let $G$ be a simple brick, and let e be a strictly thin edge of index three such that the retract of $G-e$ is an odd wheel $W_{2 k+1}$, where $k \in\{2,3\}$. If $k=2$ then $G$ is not Birkhoff-von Neumann. If $k=3$ then either $G$ is not Birkhoff-von Neumann, or $G$ is not PM-compact, possibly both.

Proof: We adopt Notation 3.11. The shrinking of $\left\{u_{2}, u_{0}, u_{1}, v_{0}, v_{2}\right\}$ results in the contraction vertex of $H$ which has degree at least five, whence the hub of $H$ is indeed the contraction vertex. Let $Q$ denote the rim of $H$, and label its vertices in cyclic order as follows: $\left(w_{0}, w_{1}, w_{2}, \ldots, w_{2 k}, w_{0}\right)$. 
First suppose that $k=2$. Since $|V(Q)|=5$, each of $u_{2}$ and $v_{2}$ has precisely two neighbours in $V(Q)$, and $u_{1}$ has only one neighbour in $V(Q)$. Adjust notation so that $w_{0} u_{1}, w_{1} u_{2} \in E(G)$. See Figure 8. Observe that only one of the vertices in $\left\{w_{2}, w_{3}, w_{4}\right\}$ is a neighbour of $u_{2}$, and the remaining two are neighbours of $v_{2}$. In each case, we present an odd conformal bicycle $\left(C_{1}, C_{2}\right)$. Let $C_{1}:=\left(u_{1}, u_{0}, v_{0}, u_{1}\right)$. If $w_{2} u_{2} \in E(G)$, then let $C_{2}:=\left(w_{2}, u_{2}, w_{1}, w_{2}\right)$. If $w_{3} u_{2} \in$ $E(G)$, then let $C_{2}:=\left(w_{3}, u_{2}, w_{1}, w_{0}, w_{4}, w_{3}\right)$. If $w_{4} u_{2} \in E(G)$, then let $C_{2}:=\left(w_{2}, v_{2}, w_{3}, w_{2}\right)$. Thus, when $k=2, G$ is not Birkhoff-von Neumann. This proves the first part.

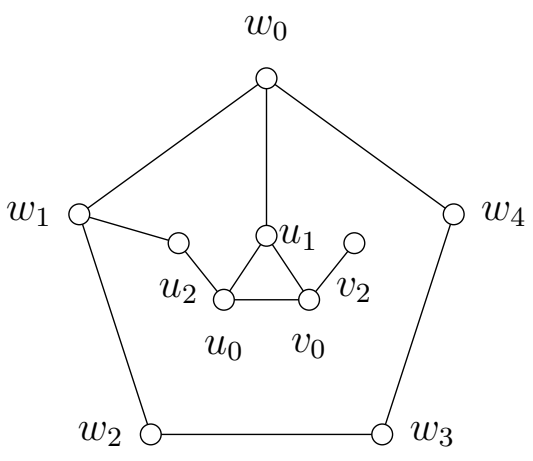

Figure 8: Illustration for the proof of Proposition 3.17 when $k=2$

Now suppose that $k=32$ First, let us assume that either $u_{2}$, or $v_{2}$, has two neighbours that appear consecutively on the $\operatorname{rim} Q$. Adjust notation so that $u_{2} w_{6}, u_{2} w_{0} \in E(G)$. See Figure 9(a). Observe that, if $v_{2}$ is a neighbour of any vertex in $\left\{w_{1}, w_{3}, w_{5}\right\}$ then the two triangles $\left(w_{0}, u_{2}, w_{6}, w_{0}\right)$ and $\left(u_{1}, u_{0}, v_{0}, u_{1}\right)$ constitute an odd conformal bicycle, and we are done. So we may assume that $v_{2}$ is not a neighbour of any vertex in $\left\{w_{1}, w_{3}, w_{5}\right\}$, whence $v_{2} w_{2}, v_{2} w_{4} \in E(G)$. See Figure 9(b). Now, $w_{1}$ is either a neighbour of $u_{2}$ or of $u_{1}$. If $w_{1} u_{2} \in E(G)$, then the two triangles $\left(w_{1}, u_{2}, w_{0}, w_{1}\right)$ and $\left(u_{1}, u_{0}, v_{0}, u_{1}\right)$ constitute an odd conformal bicycle. If $w_{1} u_{1} \in E(G)$, then the 6 -cycle $\left(w_{1}, u_{1}, v_{0}, u_{0}, u_{2}, w_{0}, w_{1}\right)$ and the 4 -cycle $\left(w_{2}, v_{2}, w_{4}, w_{3}, w_{2}\right)$ constitute an even conformal bicycle. Thus, in either case, we are done.

Next, let us assume that $u_{2}$ has two neighbours $w_{i}$ and $w_{i+2}$ on the rim and that $v_{2}$ is a neighbour of $w_{i+1}$, where subscript arithmetic is done modulo 7. See Figure 9(c) for an example. Then the triangle $\left(u_{1}, u_{0}, v_{0}, u_{1}\right)$ and the 7 -cycle $Q+w_{i} u_{2}+w_{i+2} u_{2}-w_{i} w_{i+1}-$ $w_{i+1} w_{i+2}$ constitute an odd conformal bicycle, and we are done.

Henceforth, we may assume the following. Neither $u_{2}$ nor $v_{2}$ has two neighbours that appear consecutively on the $\operatorname{rim} Q$. Furthemore, if $u_{2} w_{i}, u_{2} w_{i+2} \in E(G)$ then $u_{1} w_{i+1} \in E(G)$. Likewise, if $v_{2} w_{i}, v_{2} w_{i+2} \in E(G)$ then $u_{1} w_{i+1} \in E(G)$.

Let us assume that $u_{2}$ has two neighbours $w_{i}$ and $w_{i+2}$ on the rim. Adjust notation so that $u_{2} w_{6}, u_{2} w_{1} \in E(G)$. It follows from the aforementioned assumptions that $u_{1} w_{0} \in E(G)$. See Figure 9(d). Furthermore, at most one of $w_{3}$ and $w_{4}$ is a neighbour of $v_{2}$. Since $v_{2}$

\footnotetext{
${ }^{2}$ Unlike the $k=2$ case, there are too many possibilities for the graph $G$. More precisely, there are 46 nonisomorphic possibilities as can be checked by computations.
} 


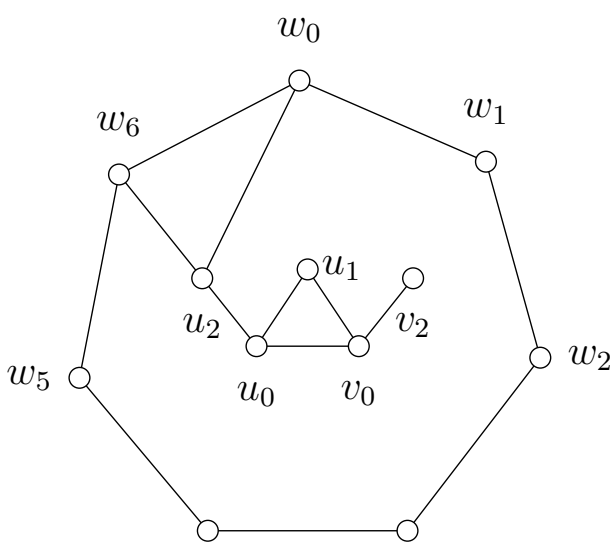

(a)

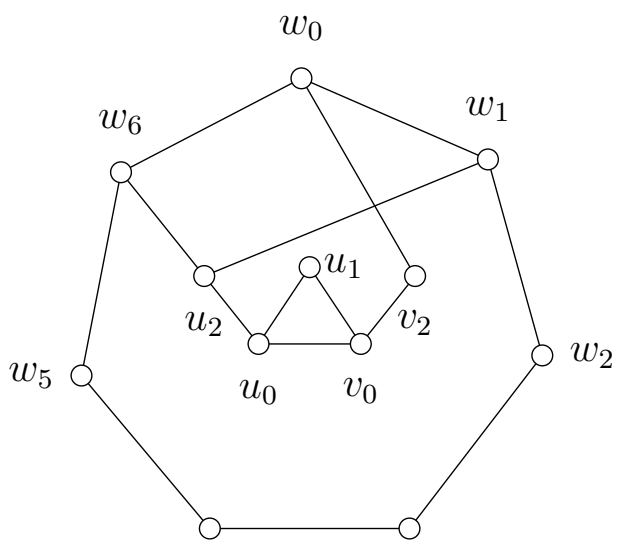

(c)

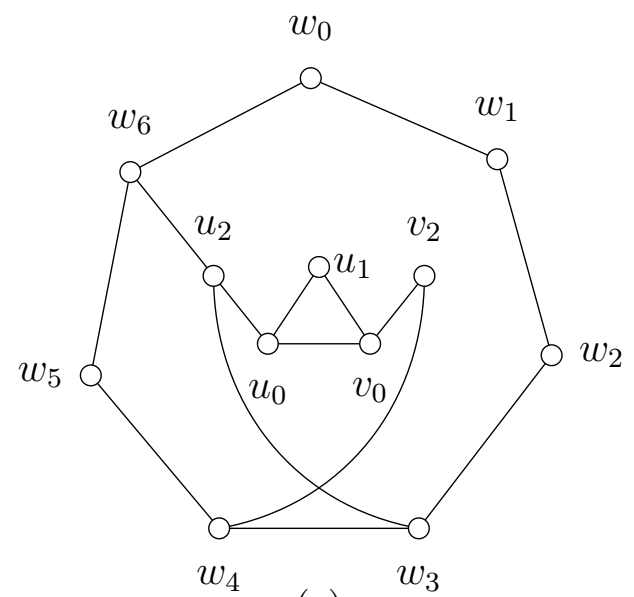

(e)

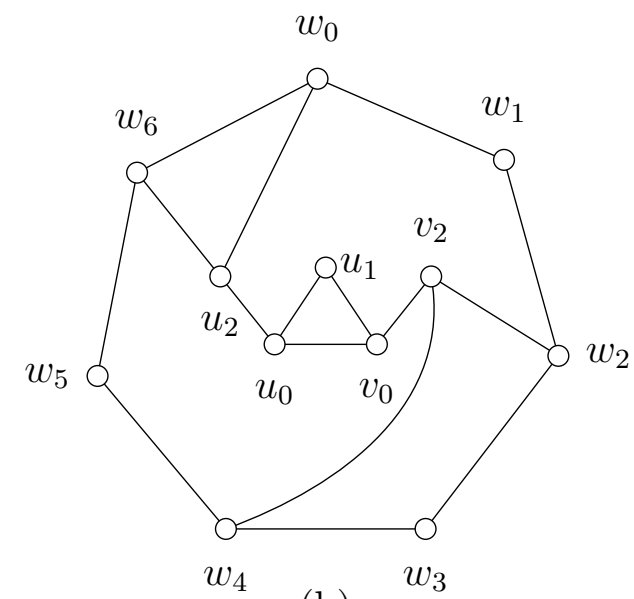

(b)

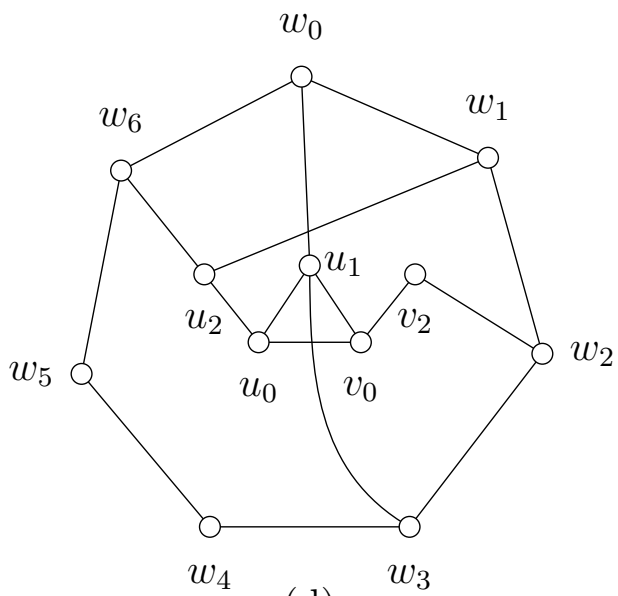

(d)

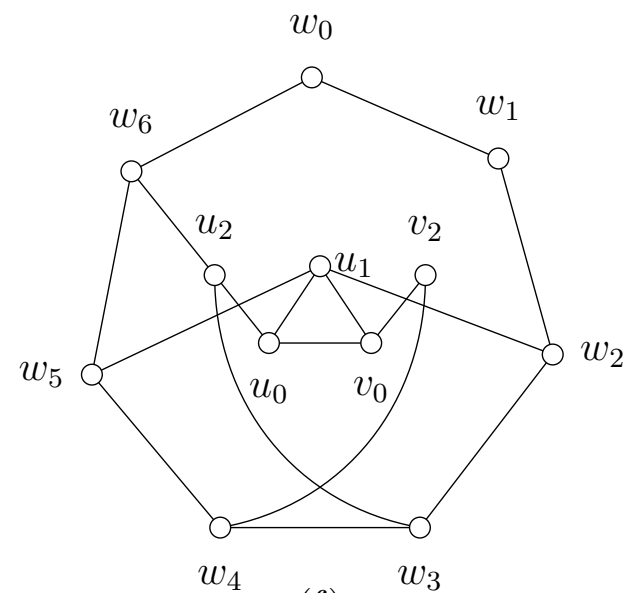

(f)

Figure 9: Illustration for the proof of Proposition 3.17 when $k=3$ 
has at least two neighbours in $V(Q)$, at least one of $w_{2}$ and $w_{5}$ is a neighbour of $v_{2}$. By symmetry, we may adjust notation so that $v_{2} w_{2} \in E(G)$. Consequently, $w_{3}$ is not a neighbour of $v_{2}$. Furthermore, since $w_{1} u_{2}, w_{2} v_{2} \in E(G), w_{3}$ is not a neighbour of $u_{2}$. Thus $w_{3} u_{1} \in$ $E(G)$. Observe that the 6 -cycle $\left(w_{3}, u_{1}, u_{0}, v_{0}, v_{2}, w_{2}, w_{3}\right)$ and the 4-cycle $\left(w_{0}, w_{6}, u_{2}, w_{1}, w_{0}\right)$ constitute an even conformal bicycle, and we are done.

Henceforth we may assume the following. If either $u_{2}$, or $v_{2}$, has two neighbours $w_{i}, w_{j} \in$ $V(Q)$ then the distance between $w_{i}$ and $w_{j}$ on the rim is exactly three. Consequently, $u_{2}$ and $v_{2}$ are cubic vertices of $G$.

Adjust notation so that $u_{2} w_{3}, u_{2} w_{6} \in E(G)$. See Figure 9(e). It follows from our assumption that at least one of $w_{4}$ and $w_{5}$ is a neighbour of $v_{2}$. By symmetry, we may adjust notation so that $w_{4} v_{2} \in E(G)$. Consequently, $w_{2}$ and $w_{5}$ are both neighbours of $u_{1}$. See Figure 9(f). Now the two 6-cycles $\left(w_{2}, u_{1}, w_{5}, w_{6}, w_{0}, w_{1}, w_{2}\right)$ and $C_{2}:=\left(w_{3}, u_{2}, u_{0}, v_{0}, v_{2}, w_{4}, w_{3}\right)$ constitute an even conformal bicycle.

Thus, in every case, we have found a conformal bicycle in $G$, whence either $G$ is not Birkhoff-von Neumann, or $G$ is not PM-compact, possibly both. This proves the second part of Proposition 3.17 .

Lemma 3.18 Let $G$ be a simple brick, and let e be a strictly thin edge of index three such that the retract of $G-e$ is an odd wheel $W_{2 k+1}$ for some $k \geq 4$. Then $G$ has distinct edges $f_{1}$ and $f_{2}$, each of which is distinct from e, such that the following hold:

(i) $f_{1}$ is strictly thin of index one in $G$; let $H_{1}$ denote the retract of $G-f_{1}$;

(ii) $f_{2}$ is strictly thin of index zero in $H_{1}$; let $H_{2}:=H_{1}-f_{2}$;

(iii) $e$ is strictly thin of index three in $\mathrm{H}_{2}$, and the retract of $\mathrm{H}_{2}-e$ is the odd wheel $W_{2 k-1}$.

Proof: We adopt Notation 3.11. The shrinking of $\left\{u_{2}, u_{0}, u_{1}, v_{0}, v_{2}\right\}$ results in the contraction vertex of $H$ which has degree at least five, whence the hub of $H$ is indeed the contraction vertex. We let $Q$ denote the rim of $H$, and we label its vertices in cyclic order as follows: $\left(w_{0}, w_{1}, \ldots, w_{2 k}, w_{0}\right)$. Thus $Q$ is a cycle of length $2 k+1$ in $G$. We let $S:=\left\{u_{2}, u_{1}, v_{2}\right\}$. Since $k \geq 4$, the sum of the degrees of members of $S$ is at least 13 ; this proves the following.

3.18.1 At most two members of $S$ are cubic, and at least one member of $S$ has degree five or more.

Each vertex in $V(Q)$ is a neighbour of exactly one vertex in $S$. This helps us define a function $\sigma: V(Q) \rightarrow S$ as follows. For each $w_{i} \in V(Q)$, let $\sigma\left(w_{i}\right)$ denote the unique vertex in $S$ that is a neighbour of $w_{i}$. Now we shall establish the following; it will help us in locating a pair of edges $f_{1}$ and $f_{2}$ that satisfy statements (i), (ii) and (iii).

3.18.2 There exists $w_{i} \in V(Q)$ such that:

(a) $\sigma\left(w_{i}\right)$ has degree four or more in $G$, 
(b) $\sigma\left(w_{i-1}\right) \neq 3\left(w_{i+1}\right)$, and

(c) at least one of $\sigma\left(w_{i-1}\right)$ and $\sigma\left(w_{i+1}\right)$ has degree four or more in $G-\sigma\left(w_{i}\right) w_{i}$,

Proof: By 3.18.1, the set $S$ has zero, one or two cubic vertices.

First we consider the case: $S$ has zero cubic vertices. Since $Q$ is an odd cycle, and since each member of $S$ has at least one neighbour in $V(Q)$, it follows that there exists $w_{i} \in V(Q)$ such that $\sigma\left(w_{i-1}\right) \neq \sigma\left(w_{i+1}\right)$; whence at least one of $\sigma\left(w_{i-1}\right)$ and $\sigma\left(w_{i+1}\right)$ is different from $\sigma\left(w_{i}\right)$; this yields the desired conclusion.

Now we consider the case: $S$ has precisely one cubic vertex, say $z$. The graph $Q-N(z)$ has at most two components; each of these components is a path; at least one of them, say $P$, has four or more vertices. Adjust notation so that $P:=\left(w_{i}, w_{i+1}, \ldots, w_{j-1}, w_{j}\right)$ where $i<j$. Note that $w_{i}$ and $w_{j}$ both satisfy statements (a) and (b). Furthermore, if either $\sigma\left(w_{i}\right)$ has degree five or more, or if $\sigma\left(w_{i}\right) \neq \sigma\left(w_{i+1}\right)$, then $w_{i}$ satisfies statement (c). Likewise, if $\sigma\left(w_{j}\right)$ has degree five or more, or if $\sigma\left(w_{j}\right) \neq \sigma\left(w_{j-1}\right)$, then $w_{j}$ satisfies statement (c). Now suppose that none of these conditions hold; in other words, $\sigma\left(w_{i}\right)=\sigma\left(w_{i+1}\right)$ and $\sigma\left(w_{j}\right)=\sigma\left(w_{j-1}\right)$ are vertices of degree precisely four. Observe that $\sigma\left(w_{i}\right) \neq \sigma\left(w_{j}\right)$ - since otherwise the degree of $\sigma\left(w_{i}\right)$ is five or more. Consequently, $S=\left\{z, \sigma\left(w_{i}\right), \sigma\left(w_{j}\right)\right\}$; this contradicts 3.18.1.

Finally, we consider the case: $S$ has precisely two cubic vertices, say $y$ and $z$. The graph $Q-N(y)-N(z)$ has at most four components; each of these components is a path; at least one of them, say $P$, has two or more vertices. We let $w_{i}$ denote an end of $P$. By 3.18.1, the vertex $\sigma\left(w_{i}\right)$ has degree at least five; note that $w_{i}$ satisfies statements $(a)$, (b) and (c).

This completes the proof of 3.18 .2 .

We invoke 3.18.2, and adjust notation so that $\sigma\left(w_{1}\right)$ has degree four or more (in $G$ ), $\sigma\left(w_{0}\right) \neq \sigma\left(w_{2}\right)$ and $\sigma\left(w_{2}\right)$ has degree four or more in $G-\sigma\left(w_{1}\right) w_{1}$. We let $f_{1}:=\sigma\left(w_{1}\right) w_{1}$ and $f_{2}:=\sigma\left(w_{2}\right) w_{2}$.

Observe that only one end of $f_{1}$, namely $w_{1}$, is cubic. We let $H_{1}$ denote the retract of $G-f_{1}$. Note that $H_{1}$ is simple since $\sigma\left(w_{0}\right) \neq \sigma\left(w_{2}\right)$, and that its unique contraction vertex is incident with the edge $f_{2}$. Also, since $\sigma\left(w_{2}\right)$ has degree at least four in $G-f_{1}$, each end of $f_{2}$ is noncubic in $H_{1}$. We let $H_{2}:=H_{1}-f_{2}$. Observe that both ends of $e$ are cubic in $H_{2}$, and that the retract of $H_{2}-e$ is indeed the odd wheel $W_{2 k-1}$. By Theorem 3.3. $H_{2}$ is a (simple) brick, and $e$ is a strictly thin edge of index three in $H_{2}$. Since $H_{1}$ is obtained from $H_{2}$ by adding an edge, $H_{1}$ is also a (simple) brick and $f_{2}$ a strictly thin edge of index zero in $H_{1}$. Consequently, $f_{1}$ is a strictly thin edge of index one in $G$. This completes the proof of Lemma 3.18.

We now have the following consequence by repeatedly applying Lemma 3.18 ,

Corollary 3.19 Let $G$ be a simple brick, and let e be a strictly thin edge of index three such that the retract of $G-e$ is an odd wheel $W_{2 k+1}$ where $k \geq 3$. Then there exists a sequence

\footnotetext{
${ }^{3}$ All of the subscript arithmetic is done modulo $2 k+1$.
} 
$G_{1}, G_{2}, \ldots, G_{r}$ of simple bricks such that (i) $G_{1}$ is $W_{7}$; (ii) $G_{r}=G$; (iii) for $2 \leq i \leq r, G_{i}$ has a strictly thin edge $e_{i}$ such that $G_{i-1}$ is the retract of $G_{i}-e_{i}$; and (iv) $e_{2}$ is of index three in $G_{2}$.

\subsection{Proof of Theorem 2.4}

Proof of Theorem 2.4: Let $G$ be a brick that is Birkhoff-von Neumann as well as PM-compact. We induct on the number of edges.

First suppose that either $G$ is not simple and let $e$ denote a multiple edge of $G$, or otherwise $G$ is a simple brick that has a strictly thin edge $e$ of index zero. In either case, $G-e$ is a smaller brick that is also Birkhoff-von Neumann and PM-compact. By the induction hypothesis, $G-e$ is one of the graphs listed in Theorem 2.4. The result follows from Propositions 3.9 and 3.10 .

Now suppose that $G$ is a simple brick that is free of strictly thin edges of index zero. If $G$ is a Norine-Thomas brick then we are done by Proposition 3.8. Now suppose that $G$ is not a Norine-Thomas brick. In this case, we invoke Theorem 3.5, whence $G$ has a strictly thin edge $e$ of index at least one. We let $H$ denote the retract of $G-e$. Thus $H$ is a simple brick that is Birkhoff--von Neumann as well as PM-compact. By the induction hypothesis, $H$ is either an odd wheel, or it is the Murty graph. We consider three cases depending on the index of $e$.

Case 1: Edge $e$ is of index one.

It follows from Proposition 3.4 that $H$ has just one contraction vertex, and its degree is at least four. By the induction hypothesis, $H$ is either an odd wheel of order at least six, or $H$ is the Murty graph. In the latter case, we arrive at a contradition by invoking Proposition 3.12 .

Now suppose that $H$ is an odd wheel. Corollary 3.15 implies that there exists a sequence $G_{1}, G_{2}, \ldots, G_{r}$ of simple bricks such that (i) $G_{1}=W_{5}$; (ii) $G_{r}=G$; (iii) for $2 \leq i \leq r, G_{i}$ has a strictly thin edge $e_{i}$ such that $G_{i-1}$ is the retract of $G_{i}-e_{i}$; (iv) $e_{2}$ is of index one in $G_{2}$; and (v) if $r \geq 3$ then $e_{3}$ is of index zero in $G_{3}$.

Note that all of the bricks $G_{1}, G_{2}, \ldots, G_{r}$ are Birkhoff-von Neumann and PM-compact. Since $G_{1}$ is $W_{5}$, Proposition 3.13 implies that $G_{2}$ is indeed the Murty graph. If $r=2$ then we are done. Now suppose that $r \geq 3$. Thus $G_{3}$ is obtained from $G_{2}$ by adding an edge joining two nonadjacent vertices. We invoke Proposition 3.10 to arrive at a contradiction.

Case 2: Edge $e$ is of index two.

It follows from Proposition 3.4 that $H$ has two contraction vertices, each of which has degree at least four. By the induction hypothesis, $H$ is in fact the Murty graph. We invoke Proposition 3.16 to arrive at a contradiction.

Case 3: Edge $e$ is of index three.

It follows from Proposition 3.4 that $H$ has just one contraction vertex, and its degree is at least five. By the induction hypothesis, $H$ is in fact an odd wheel of order at least six. If $H=W_{5}$, we arrive at a contradiction by invoking Proposition 3.17. 
Now suppose that $H=W_{2 k+1}$ where $k \geq 3$. Corollary 3.19 implies that there exists a sequence $G_{1}, G_{2}, \ldots, G_{r}$ of simple bricks such that (i) $G_{1}=W_{7}$; (ii) $G_{r}=G$; (iii) for $2 \leq i \leq r, G_{i}$ has a strictly thin edge $e_{i}$ such that $G_{i-1}$ is the retract of $G_{i}-e_{i}$; and (iv) $e_{2}$ is of index three in $G_{2}$.

Note that all of the bricks $G_{1}, G_{2}, \ldots, G_{r}$ are Birkhoff-von Neumann and PM-compact. However, since $G_{1}$ is $W_{7}$, Proposition 3.17 implies that either $G_{2}$ is not Birkhoff-von Neumann, or $G_{2}$ is not PM-compact, possibly both. We thus have a contradiction.

This completes the proof of Theorem 2.4.

\subsection{An infinite family}

Thus far we have given a complete characterization of matching covered graphs that are Birkhoff-von Neumann as well as PM-compact. In a recent paper, Lucchesi, Carvalho, Kothari and Murty [1] showed that the problem of characterizing Birkhoff-von Neumann bricks 4 is equivalent to the problem of characterizing $\overline{C_{6}}$-free bricks. In the same work, they present two infinite families of Birkhoff-von Neumann bricks; see [11, Figures 7 and 8]. The members of these families, except for the Murty graph, are not PM-compact (as expected).

Now we present a family of PM-compact bricks that are not Birkhoff-von Neumann. For $k \geq 2$, let $W_{2 k+1}$ denote the odd wheel and let $Q$ denote its rim. We label the vertices of $Q$, in cyclic order, as follows: $\left(w_{0}, w_{1}, \ldots, w_{2 k}\right)$. We obtain a new graph from $W_{2 k+1}$ as follows.

First split the hub of $W_{2 k+1}$ into three pairwise nonadjacent vertices $u_{2}, u_{1}$ and $v_{2}$ and distribute the spokes of the wheel so that:

- $v_{2}$ is adjacent to $w_{0}$ and $w_{4}$;

- $u_{1}$ is adjacent to $w_{2}$; and

- $u_{2}$ is adjacent to the remaining $2 k-2$ vertices; that is, to $w_{1}$, to $w_{3}$, and if $k \geq 3$ then to each of $w_{5}, w_{6}, \ldots, w_{2 k}$.

Now add two new vertices $u_{0}$ and $v_{0}$, and the following edges: $u_{0} u_{2}, u_{0} u_{1}, u_{0} v_{0}, v_{0} u_{1}, v_{0} v_{2}$. The graph obtained in this manner is denoted by $\mathcal{P}_{2 k+1}$. In Figure 10, we show the brick $\mathcal{P}_{7}$. Observe that the retract of $\mathcal{P}_{2 k+1}-u_{0} v_{0}$ is the odd wheel $W_{2 k+1}$. It follows from Theorem 3.3 that $\mathcal{P}_{2 k+1}$ is indeed a brick.

Proposition 3.20 For $k \geq 2$, the brick $\mathcal{P}_{2 k+1}$ is PM-compact, but it is not Birkhoff-von Neumann.

Proof: Let $C_{1}:=\left(v_{0}, u_{1}, u_{0}, v_{0}\right)$. If $k=2$ then let $C_{2}:=\left(w_{0}, v_{2}, w_{4}, w_{0}\right)$; otherwise let $C_{2}:=\left(w_{0}, v_{2}, w_{4}, w_{5}, w_{6}, \ldots, w_{2 k}, w_{0}\right)$. Observe that $\left(C_{1}, C_{2}\right)$ is an odd conformal bicycle. Thus $\mathcal{P}_{2 k+1}$ is not Birkhoff-von Neumann.

\footnotetext{
${ }^{4}$ As mentioned earlier, this class is the same as the class of solid bricks.
} 


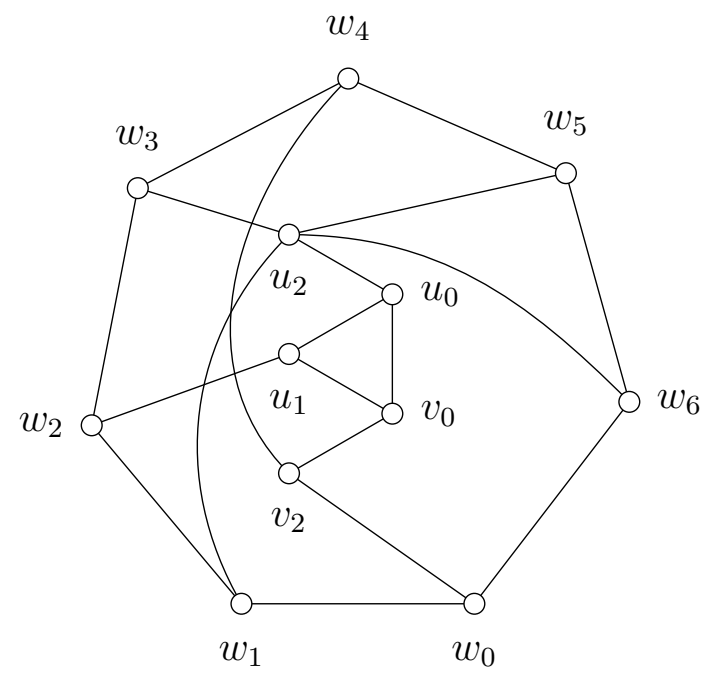

Figure 10: The brick $\mathcal{P}_{7}$

Now let $G:=\mathcal{P}_{2 k+1}$ for some $k \geq 2$, and suppose (for the sake of contradiction) that $G$ is not PM-compact. Consequently, $G$ has an even conformal bicycle $\left(C_{1}, C_{2}\right)$. Observe that $G-v_{2}-u_{2}$ is free of even cycles. Thus one of $C_{1}$ and $C_{2}$ contains $v_{2}$ whereas the other contains $u_{2}$. Adjust notation so that $v_{2} \in V\left(C_{1}\right)$ and $u_{2} \in V\left(C_{2}\right)$.

We let $D:=\partial\left(\left\{v_{0}, u_{1}, u_{0}\right\}\right)$. Note that $D$ is a 3 -cut. Since $G$ is simple, we may also view a cycle as a set of edges without any ambiguity. We will use the fact that $\left|C_{1} \cap D\right|$ and $\left|C_{2} \cap D\right|$ are both even. Since $u_{2} \in V\left(C_{2}\right)$, we infer that either $C_{1} \cap D=\left\{v_{2} v_{0}, u_{1} w_{2}\right\}$ or otherwise $C_{1} \cap D=\emptyset$.

First consider the case in which $C_{1} \cap D=\emptyset$. Thus $v_{2} w_{0}, v_{2} w_{4} \in C_{1}$. Since $C_{1}$ is an even cycle, $C_{1}$ is now uniquely determined; more specifically, $C_{1}:=\left(w_{0}, v_{2}, w_{4}, w_{3}, w_{2}, w_{1}, w_{0}\right)$. Now we observe that $C_{2} \cap D=\emptyset$. However, $u_{2} \in V\left(C_{2}\right)$. Consequently, the triangle $\left(v_{0}, u_{1}, u_{0}, v_{0}\right)$ is a component of $G-V\left(C_{1}\right)-V\left(C_{2}\right)$. Thus the pair $\left(C_{1}, C_{2}\right)$ is not conformal, contrary to our assumption.

Now consider the case in which $C_{1} \cap D=\left\{v_{2} v_{0}, u_{1} w_{2}\right\}$. It follows that $C_{2} \cap D=\emptyset$. If $u_{0} \notin V\left(C_{1}\right)$ then $u_{0}$ is an isolated vertex in $G-V\left(C_{1}\right)-V\left(C_{2}\right)$, contrary to our assumption. Thus $u_{0} \in V\left(C_{1}\right)$, whence $v_{0} u_{0}, u_{1} u_{0} \in C_{1}$. Note that $C_{1}$ contains exactly one of $v_{2} w_{0}$ and $v_{2} w_{4}$. However, these two cases are symmetric. Adjust notation so that $v_{2} w_{0} \in C_{1}$. Since $C_{1}$ is an even cycle, $C_{1}$ is again determined uniquely; more specifically, $C_{1}:=\left(w_{0}, v_{2}, v_{0}, u_{0}, u_{1}, w_{2}, w_{3}, w_{4}, \ldots, w_{2 k}, w_{0}\right)$. Now observe that $G-V\left(C_{1}\right)$ has precisely two vertices - namely, $u_{2}$ and $w_{1}$. This contradicts the existence of $C_{2}$.

Since each case leads us to a contradiction, we conclude that $\mathcal{P}_{2 k+1}$ is indeed PM-compact. This completes the proof of Proposition 3.20,

Acknowledgements: We would like to extend our gratitude to one of the anonymous referees who provided detailed feedback and also observed a bug in the "proof" of Lemma 3.18 in the original manuscript. 


\section{References}

[1] E. Balas. Integer and fractional matchings. In P. Hansen, editor, Studies on Graphs and Discrete Programming, volume 59, pages 1-13. North Holland Mathematics Studies, 1981.

[2] M. L. Balinksi and A. Russakoff. On the assignment polytope. SIAM Review, 16(4):516$525,1974$.

[3] M. H. Carvalho, C. L. Lucchesi, and U. S. R. Murty. On a conjecture of Lovász concerning bricks. I. The characteristic of a matching covered graph. J. Combin. Theory Ser. B, 85:94-136, 2002.

[4] M. H. Carvalho, C. L. Lucchesi, and U. S. R. Murty. The perfect matching polytope and solid bricks. J. Combin. Theory Ser. B, 92:319-324, 2004.

[5] M. H. Carvalho, C. L. Lucchesi, and U. S. R. Murty. Graphs with independent perfect matchings. J. Graph Theory, 48:19-50, 2005.

[6] M. H. Carvalho, C. L. Lucchesi, and U. S. R. Murty. How to build a brick. Discrete Math., 306:2383-2410, 2006.

[7] V. Chvátal. On certain polytopes associated with graphs. J. Combin. Theory Ser. B, 18:138-154, 1975.

[8] J. Edmonds, L. Lovász, and William R. Pulleyblank. Brick decomposition and the matching rank of graphs. Combinatorica, 2:247-274, 1982.

[9] N. Kothari and U. S. R. Murty. $K_{4}$-free and $\overline{C_{6}}$-free planar matching covered graphs. J. Graph Theory, 82(1):5-32, 2016.

[10] L. Lovász. Matching structure and the matching lattice. J. Combin. Theory Ser. B, 43:187-222, 1987.

[11] C. L. Lucchesi, M. H. Carvalho, N. Kothari, and U. S. R. Murty. On two unsolved problems concerning matching covered graphs. SIAM J. Discrete Math., 32(2):14781504, 2018.

[12] S. Norine and R. Thomas. Generating bricks. J. Combin. Theory Ser. B, 97:769-817, 2007.

[13] X. Wang, Y. Lin, M. H. Carvalho, C. L. Lucchesi, G. Sanjith, and C. H. C. Little. A characterization of PM-compact bipartite and near-bipartite graphs. Discrete Math., 313:772-783, 2013.

[14] X. Wang, W. Shang, Y. Lin, and C. L. Lucchesi. A characterization of PM-compact claw-free cubic graphs. Discrete Math. Algorithms Appl., 6(2), 2014. 
[15] X. Wang, J. Yuan, and Y. Lin. A characterization of PM-compact Hamiltonian bipartite graphs. Acta Math. Appl. Sin. Engl. Ser., 31(2):313-324, 2015. 